\title{
Monitoring Alpha-Synuclein - Tau Interactions In Vitro and In Vivo Using Bimolecular Fluorescence Complementation
}

Laura Torres-Garcia

Lund University

Joana M.P. Domingues

Lund University

Edoardo Brandi

Lund University

Caroline Haikal

Lund University

Inês C. Brás

University Medical Center Göttingen

Ellen Gerhardt

University Medical Center Göttingen

Wen Li

Lund University

Alexander Svanbergsson

Lund University

Tiago F. Outeiro

University Medical Center Göttingen

Gunnar K. Gouras

Lund University

Jia-Yi Li ( $\sigma$ jia-yi.li@med.lu.se )

Lund University

\section{Research Article}

Keywords: Parkinson's disease, Alzheimer's disease, Lewy bodies

Posted Date: October 22nd, 2021

DOI: https://doi.org/10.21203/rs.3.rs-960446/v1 
License: (c) (i) This work is licensed under a Creative Commons Attribution 4.0 International License. Read Full License 


\section{Abstract}

Parkinson's disease (PD) and Alzheimer's disease (AD) are characterized by pathological accumulation and aggregation of different amyloidogenic proteins, a-synuclein (aSyn) in PD, and amyloid- $\beta$ (A $\beta$ ) and Tau in AD. Strikingly, few PD and AD patients' brains exhibit pure pathology with most cases presenting mixed types of protein deposits in the brain. Bimolecular fluorescence complementation (BiFC) is a technique based on the complementation of two halves of a fluorescent protein, which allows direct visualization of protein-protein interactions. In the present study, we assessed the ability of aSyn and Tau to interact with each other. For in vitro evaluation, HEK293 and human neuroblastoma cells were used, while in vivo studies were performed by AAV6 injection in the substantia nigra pars compacta (SNpc) of mice and rats. We observed that the co-expression of aSyn and Tau led to the emergence of fluorescence, reflecting the interaction of the proteins in cell lines, as well as in mouse and rat SNpc. Thus, our data indicates that aSyn and Tau are able to interact with each other in a biologically relevant context, and that the BiFC assay is an effective tool for studying aSyn-Tau interactions in vitro and in different rodent models in vivo.

\section{Introduction}

Parkinson's disease (PD) is the most common movement disorder and is characterized by the pathological accumulation of a-synuclein (aSyn) in the form of Lewy bodies (LB) and Lewy neurites (LN), and by the death of dopaminergic neurons in the substantia nigra in the midbrain ${ }^{1}$. Alzheimer's disease $(A D)$ is the major form of dementia, and the accumulation of amyloid- $\beta(A \beta)$ and Tau in $A \beta$ plaques and neurofibrillary tangles (NFT), respectively, are pathological hallmarks of AD; however, Tau pathology correlates better with the cognitive decline associated with $A D^{2}$.

Genome-wide association studies (GWAS) have identified the MAPT gene, which encodes for Tau protein; together with the SNCA gene, which encodes for aSyn protein, as major risk factors for the development of $\mathrm{PD}^{3,4}$. Furthermore, in human brains co-occurrence of aSyn and Tau pathologies has been described. About $50 \%$ of PD postmortem brains show NFTs, while LBs and LNs are detected in $60 \%$ of cases with $A D^{5,6}$. Moreover, aSyn aggregates are also found in Frontotemporal dementia (FTD) 7,8 , Progressive supranuclear palsy (PSP) ${ }^{9}$ and Down's syndrome with $A D^{10,11}$. Likewise, Tau accumulation has been observed in Dementia with Lewy bodies (DLB), also called Diffuse Lewy body disease (DLBD) ${ }^{12-14}$; and Parkinson's disease dementia (PDD) ${ }^{15,16}$. In particular, the co-occurrence of aSyn and Tau in amygdala has been reported in AD, PD, Down's syndrome and DLB $12,13,17,18$.

Animal studies have shown that although Tau does not seem to be involved in aSyn spreading in the brain ${ }^{19}$, it plays an important role in synuclein pathology. In mice expressing human PD mutant A53T aSyn, Tau is required for the deficits in learning, memory and synaptic plasticity. A53T aSyn appears to induce Tau phosphorylation by glycogen synthase kinase-3 $\beta$ (GSK3 $\beta$ ) activation, which drives the missorting of Tau to dendritic compartments and an increase in the internalization of AMPA receptors 
(AMPARs), in the absence of clear neuropathology ${ }^{20,21}$. Additionally, rats virally expressing human Tau (WT or P301L mutant) in substantia nigra pars compacta (SNpc) show vulnerability of dopaminergic neurons to Tau-induced neurodegeneration, and human Tau expression leads to a stronger amphetamineinduced rotational behavior than the expression of human aSyn (A30P or A53T mutant) alone in the same area ${ }^{22}$. Similar observations have been described in a mouse model carrying the human K369I Tau mutation, in which impairment in the anterograde axonal transport induced by Tau results in loss of dopaminergic neurons and a Parkinsonian-like phenotype ${ }^{23}$.

Moreover, aSyn appears to be essential for the phosphorylation of Tau in primary neurons after treatment with MPTP, a potent inducer of parkinsonism ${ }^{1}$. Tau co-localizes and interacts with aSyn in aSyn aggregates ${ }^{24}$ and both are found together by immunofluorescence microscopy in the axons of cultured primary hippocampal neurons ${ }^{25}$, and in excitatory pre-synaptic terminals ${ }^{26}$. Furthermore, cell free work has shown that aSyn is able to interact with Tau by binding of its $\mathrm{C}$-terminal region to the microtubule binding domain (MTBD) of Tau ${ }^{25,27}$. Thus, there is a growing body of evidence supporting that aSyn and Tau are able to interact and influence each other. However, how the interaction between aSyn and Tau enhances neurodegeneration is still poorly understood.

Bimolecular Fluorescence Complementation (BiFC) is a technique widely used to examine protein-protein interactions. It is based on the structural complementation of two halves of a fluorescent protein, allowing direct visualization of interacting proteins. In neurodegenerative diseases, this technique has

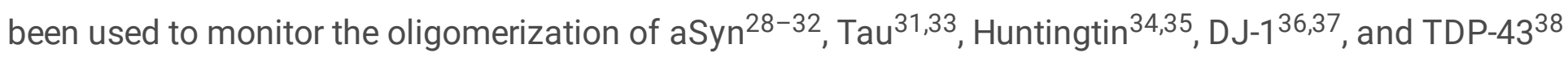
in vitro, as well as to study self-interaction of $\mathrm{aSyn}^{39,40}$ and $\mathrm{Tau}^{41}$ in vivo.

The aim of the present study was to assess the ability of aSyn and Tau to interact with each other, and to evaluate whether BiFC is a suitable technique to monitor aSyn-Tau interaction in vitro and in vivo. We concluded that aSyn and Tau are able to interact with each other and that the BiFC assay is an effective tool to study aSyn-Tau interaction in vitro and in vivo, providing a valuable tool for examining the pathological and physiological consequences of aSyn-Tau interaction, and facilitating the screening of potential drugs that could boost or inhibit this interaction.

\section{Results}

\section{aSyn-Tau interaction in cell lines}

The BiFC assay was chosen as a tool to evaluate aSyn-Tau interaction. aSyn (WT) and Tau (WT and P301L) were expressed fused to one of the halves of the Venus protein. After interaction of the two proteins of interest, the presence of both Venus halves in close proximity induces a conformational change in their structure that leads to their complementation and the emission of fluorescence that can be detected under a fluorescence microscope (Figure 1A). 
To examine whether aSyn-Tau interaction can be detected by the BiFC assay, we (co-) transfected HEK293 cells with different combinations of the BiFC constructs (Table S1). Single transfection of fulllength Venus allowed us to determine the maximum level of Venus signal, whereas single transfection of aSyn-Venus confirmed that full-length Venus is able to emit fluorescence when linked to aSyn (Figure 1B). On the other hand, co-transfection of the Venus halves ( $\mathrm{VN}$ - and -VC) without aSyn nor Tau linked proved that the Venus halves were not able to spontaneously complement with each other in the absence of aSyn and Tau (Figure 1B). Similar results were obtained when VN-aSyn or VN-Tau were co-expressed with the empty-VC half. However, the expression of the empty $\mathrm{VN}$ - half together with aSyn-VC or Tau-VC gave rise to weak fluorescent signal (Figure S1A).

Next, we used the BiFC assay to assess the self-interactions of aSyn, Tau WT and Tau P301L. HEK293 cells were co-transfected with paired BiFC constructs: VN-aSyn + aSyn-VC, VN-Tau WT + Tau WT-VC and VN-Tau P301L + Tau P301L-VC. These co-transfections resulted in the emergence of fluorescencepositive cells (Figure 1B), reflecting the interaction of the proteins. Thus, we confirmed that selfinteractions of aSyn, Tau WT and Tau P301L can each be observed by BiFC in HEK293 cells.

To evaluate aSyn-Tau (WT and P301L) interactions, HEK293 cells were co-transfected with aSyn and Tau (WT or P301L) linked to complementary Venus halves: VN-aSyn + Tau WT-VC, VN-Tau WT + aSyn-VC, VNaSyn + Tau P301L-VC and VN-Tau P301L + aSyn-VC. In all the combinations, we observed the complementation of the Venus protein, reflecting the interaction of aSyn and Tau (Figure 1C). The fluorescence obtained by complementation of the Venus protein was similar to the levels observed when evaluating the self-interactions of aSyn, Tau WT and Tau P301L. This demonstrates that aSyn-Tau (WT and $\mathrm{P} 301 \mathrm{~L}$ ) interaction can be monitored in vitro by BiFC.

Prior work has shown differences in the levels of expression of the different BiFC constructs. To determine the protein levels upon transfection with the BiFC constructs in our study, HEK293 cells were co-transfected with different combinations of the BiFC constructs and $24 \mathrm{~h}$ post-transfection aSyn and Tau levels were evaluated. We observed that the protein levels of aSyn and Tau were higher when they were linked to the VN- half than when linked to the -VC half (Figure 1D and S1B).

To test whether aSyn-Tau interaction can also be observed in a neuron-like cell, we (co-) transfected SHSY5Y (human neuroblastoma) cells with the different combinations of the BiFC constructs (Table S1). As observed in HEK293 cells, the single transfection of Venus led to full Venus fluorescence, while aSynVenus expression demonstrated the ability of Venus to emit fluorescence when linked to aSyn (Figure 1A). Additionally, the co-transfection of VN-, VN-aSyn, VN-Tau or VN-Tau P301L together with the empty $V C$ half showed no fluorescence, whilst we observed some fluorescence when co-expressing the empty VN- half together with aSyn-VC, Tau-VC or Tau P301L-VC (Figure 2A and S2A). In SH-SY5Y cells, aSyn, Tau WT and Tau P301L were also able to self-interact (Figure 2A), and we were able to detect complementation of Venus when aSyn and Tau (WT or P301L) were co-transfected (Figure 2B). This shows that aSyn-Tau (WT and P301L) interaction can be observed in vitro by BiFC in a human neuroblastoma cell line. 
To explore the dynamic processes of the interaction, Venus complementation in SH-SY5Y cells was examined by time-lapse, live-cell imaging every $30 \mathrm{~min}$ after transfection for $24 \mathrm{~h}$. For each condition, a field of view (FOV) was chosen and tracked, and changes in fluorescence intensity over time in the cells within the FOV were measured. We observed initial fluorescence signal at different time points between conditions. Full-length Venus was the first in emitting fluorescence $(\sim 7.5 \mathrm{~h})$, while fluorescence of aSynVenus took a longer time to appear $(\sim 11 \mathrm{~h})$; however, both conditions showed similar intensity over time. Self-Interactions of aSyn, Tau WT, and Tau P301L showed similar initiation times (9-11 h) and progression, with the aSyn coupling exhibiting the strongest complemented fluorescence (Figure S2B, upper graph). When aSyn and Tau (WT or P301L) were co-transfected, we could observe that the aSynTau P301L couplings showed earlier initiation time but lower complemented fluorescence intensity than the aSyn-Tau WT couplings (Figure S2B, lower graph).

Considering that the degree of interaction between the proteins in one cell could be influenced by the amount of plasmid(s) transfected into the cell and the levels of transgene expression, which could affect the dynamics of the interaction over time, we took a second approach to have a better picture of potential differences in the interaction between constructs. SH-SY5Y cells were (co-)transfected and after $24 \mathrm{~h}$ the percentage of fluorescent cells in relation to the total number of cells present in the FOV was quantified. This quantification showed no significant differences between aSyn, Tau WT, and Tau P301L when they were expressed by themselves or in combination. The percentage of fluorescent cells obtained after cotransfection of the different couplings was not significantly different to that obtained after single transfection of Venus or aSyn-Venus (Figure 2C), while the fluorescence emitted by VN- + -VC coupling was undetectable.

\section{Direct interaction of aSyn-Tau in mouse SNpc}

Next, we asked whether the physiological environment of the living mouse brain would enable the detection of the aSyn-Tau interaction using BiFC. For that, AAV6 carrying different BiFC constructs (Table S2) were injected into the SNpc of 10-12-week-old WT mice, and 15 weeks post-injection complemented Venus fluorescence was evaluated.

The expression of aSyn fused to full-length Venus provided the highest level of fluorescence. This expression was confined to a few neurons. As expected, and in agreement with the in vitro results, the expression of the Venus halves $(\mathrm{VN}-+-\mathrm{VC})$ not linked to interacting proteins did not produce any fluorescence in the injected area (Figure 3). Similar results were observed when VN-aSyn or VN-Tau were co-expressed with the empty - VC half. However, the expression of the empty VN- half together with aSynVC or Tau-VC gave rise to a background residual fluorescence (Figure S3). Remarkably, the co-expression of aSyn and Tau (VN-aSyn + Tau WT-VC and VN-Tau WT + aSyn-VC) led to Venus complementation (Figure 3), reflecting the interaction between aSyn and Tau in the SNpc of mice. Therefore, BiFC is shown to be a suitable technique to monitor aSyn-Tau interaction in vivo.

\section{Direct interaction aSyn-Tau in the rat SNpc}


To corroborate that the BiFC assay can be used to visualize aSyn-Tau interaction in a different animal model, we injected AAV6 carrying VN-aSyn + Tau WT-VC, the aSyn-Tau combination that yielded the strongest interaction in our mouse system, in the rat SNpc. In this case, due to the lack of toxicity induced by AAV6 and based on the results obtained in mice, we increased the viral load 10 times. After 8 weeks, Venus complementation resulting from aSyn-Tau interaction was observed (Figure 4A), confirming that the BiFC assay can be used in vivo in different rodent models.

To characterize the state of aSyn and Tau, immunohistochemistry against phosphorylated forms of aSyn and Tau was performed. AT8 immunostaining showed that Tau was substantially phosphorylated at its S202 and T205 epitopes when co-expressed together with aSyn (Figure 4A), consistent with prior work that showed that aSyn is able to induce Tau phosphorylation ${ }^{1,20,21,25}$. In addition, aSyn was also phosphorylated at its S129 epitope. Interestingly, we observed differential co-localization between complemented (BiFC) Venus, AT8-positive and pS129-positive profiles. It appeared that most of the neuronal cell bodies positive for BiFC Venus are also positive for both AT8 and pS129 (Figure 4A, white arrows), suggesting that both Tau and aSyn can be phosphorylated while they are interacting with each other. A few neuronal profiles with BiFC Venus exhibited either AT8- or pS129-positive (Figure 4A, orange arrows), implying that interacted Tau and aSyn may be phosphorylated to different extents.

Lastly, immunohistochemistry against total aSyn and total Tau was carried out to determine the distribution of the injected aSyn and Tau in the brain. Interestingly, marked propagation of the human aSyn was observed, indicative of transport from SNpc to the striatum. In contrast, the expression of human Tau remained mainly confined to the SNpc (Figure 4B).

\section{Discussion}

aSyn is a phospholipid-binding protein with a role in vesicle trafficking and neurotransmitter release ${ }^{42}$. Tau is a microtubule-associated protein involved in microtubule stabilization and axonal outgrowth ${ }^{43,44}$. Both are soluble and natively unfolded proteins ${ }^{45,46}$, that present common mechanisms of transmission ${ }^{47}$, and are characterized by their pathological aggregation in neurodegenerative disorders. aSyn and Tau accumulation leads to the formation of oligomeric and fibrillar forms. A growing body of evidence points to an intertwined interaction between aSyn and Tau in pathology. Whilst patient-derived brain tissue exhibits co-occurrence of aSyn and Tau pathology in different neurodegenerative diseases, studies carried out in animal models show that aSyn and Tau interaction increases neurotoxicity. However, how aSyn and Tau influence each other, and how their interaction enhances neurodegeneration is still poorly understood.

One of the limitations of studying the consequences of aSyn-Tau interaction is the limited number of techniques available to monitor protein interaction in vivo. We applied the BiFC assay as a potential tool to visualize aSyn-Tau interaction. BiFC has been used by several groups aiming to obtain a direct visualization of protein oligomerization due to the relevance of oligomer formation in neurodegenerative 
disorders. In synucleinopathies and tauopathies, the presence and propagation of oligomeric forms of aSyn and Tau, respectively, correlates better than fibril formation with disease pathogenesis ${ }^{42,48}$.

Previously, the direct interaction between aSyn and Tau has been demonstrated in cell free assays 25,27 . Thus, in the present study we aimed to assess the ability of aSyn and Tau to interact with each other in a biologically relevant context, and to evaluate whether BiFC is a suitable technique to monitor aSyn-Tau interaction in vitro and in vivo.

BiFC was shown to be able to track aSyn-Tau (WT and P301L) interaction in vitro in HEK293 cells and in a neuronal-like cell line, SH-SY5Y cells. After confirming the functionality of this assay to detect aSyn-Tau interaction in vitro, in vivo injections in SNpc of WT mice and rats were performed. In mice, injections with AAV6 carrying the different BiFC constructs showed that aSyn-Tau interaction could be observed in vivo by BiFC. Besides the interaction between aSyn and Tau, we observed unexpected fluorescence when empty VN- was co-expressed with aSyn-VC or Tau-VC, which did not happen when the proteins linked to the N-terminal Venus half (VN-aSyn or VN-Tau) were co-expressed with the empty -VC construct. This phenomenon has been previously reported in the literature ${ }^{30,34,49}$.

Previous studies have explored the potential of BiFC to visualize protein interactions, however, some limitations of this assay need to be kept in mind. An imbalance in the levels of expression of the BiFC constructs has been long reported in the literature $28-35,37,40,49,50$, suggesting an increase in the stability or decrease in the turnover of the VN- halves in comparison with - $\mathrm{VC}$ halves ${ }^{32}$. In our study we observed a similar phenomenon, that could potentially explain the propensity of the empty $\mathrm{VN}$ - half to interact with aSyn-VC and Tau-VC. Theoretically, the empty BiFC constructs (VN- and -VC) should not be able to complement when the interacting proteins are not present, yet, the presence of background fluorescence due to the interaction of the empty $\mathrm{VN}$ - construct has been previously described $30,34,49$.

The validity of the BiFC assay to monitor protein interaction has been tested by several groups. Robida et al. (2009) showed the ability of the BiFC assay to visualize protein interaction when this interaction had to be induced by the presence of a ligand ${ }^{49}$. In addition, point mutations in the amino acids involved in the interaction between proteins led to an inhibition of Venus complementation ${ }^{36,50-52}$. A recent study showed that even when the difference in the levels of expression of the BiFC constructs could lead to an increase in the self-aggregation of the overexpressed ones, the proteins of interest were able to physically interact with each other and this could be detected by $\mathrm{BiFC}^{32}$. Thus, the BiFC assay is suitable to determine protein-protein interactions but it is not sensitive enough to distinguish the degree of oligomerization between the interacting proteins ${ }^{32}$. This supports that the BiFC assay can be used to visualize aSyn-Tau interaction.

When VN-aSyn and Tau WT-VC AAVs were injected together in the SNpc of rats, we could observe that the co-expression of the proteins led to high levels of Tau phosphorylation at its S202 and T205 epitopes. Previous studies have shown the ability of aSyn to induce Tau phosphorylation ${ }^{1,20,21,25}$. Similarly, aSyn was phosphorylated at its pS129 epitope when co-expressed with Tau, although to a lesser extent. 
In $A D$ and $P D$, protein aggregation and neurodegeneration appear to follow a spatiotemporal

progression ${ }^{53,54}$, that suggests cell-to-cell transfer of pathology ${ }^{55-57}$. This is supported by the spreading of aSyn aggregates from the host to healthy transplanted neurons observed in postmortem tissue of PD patients ${ }^{58,59}$. In our study, after co-injection of VN-aSyn and Tau-VC in rat SNpc, we could observe spreading of human aSyn from SNpc to the striatum, in contrast, there was much less transport of human Tau, which may be caused by the different size of aSyn and Tau molecules or by different levels of expression of the proteins.

We conclude that aSyn and Tau are able to interact in a biological setting, and that the BiFC assay is an effective tool to study aSyn-Tau interaction in vitro in cells and in different rodent models in vivo, providing a new resource for examining the pathological and physiological consequences of aSyn-Tau interaction. More work needs to be done to understand the impact of this interaction, and the consequences of its inhibition and/or facilitation in neurodegeneration.

\section{Materials And Methods}

\section{Animal work: mice and rats}

C57BL/6J mice and Sprague Dawley rats were purchased from Taconic (Ejby, Denmark). Animals were randomly assigned to each group. All experiments were approved and performed following the guidelines approved by the Malmö/Lund Ethics Committee, ethical permits number: M72-2016 and M73-2016. The reporting of the animal work performed in the manuscript follows the recommendations in the ARRIVE guidelines.

\section{Generation of constructs and AVV production}

The BiFC Tau constructs, VN-Tau (WT and P301L) and Tau-VC (WT and P301L), were generated by enzymatic restriction and ligation. Venus $\mathrm{N}$-terminal (VN-) and Venus C-terminal (-VC) backbones were obtained from the VN-aSyn and aSyn-VC constructs previously described by Outeiro et al. (2008). Tau WT and Tau P301L sequences (ON4R isoform) were amplified from pRK5-EGFP-Tau (Addgene plasmid \#46904) and pRK5-EGFP-Tau P301L (Addgene plasmid \#46908) ${ }^{60}$, plasmids gift of Dr. Karen Ashe. In brief, Tau sequences were amplified by PCR (DreamTaq PCR Master Mix, Thermo Fisher Scientific). The Venus halves (VN- and -VC) were used as a backbone. The backbones and the amplified Tau sequences were cleaved by AfIII (\#ER0831, Thermo Fisher Scientific) and Xhol (\#ER0691, Thermo Fisher Scientific) restriction enzymes, and ligated by incubation with T4 DNA ligase (\#EL0014, Thermo Fisher Scientific). The generated constructs were confirmed by sequencing. From these constructs, Adeno-Associated Virus Type 6 (AAV6) were produced in the AVV Vector Lab at the MultiPark core facility at Lund University.

\section{Stereotaxic AAV Injections}

Animals were unilaterally injected with a final volume of $2 \mu$ containing different combinations of the BiFC AAVs. Vector solutions were injected using a $5 \mu \mathrm{l}$ Hamilton syringe fitted with a glass capillary. The 
injection was performed manually in the case of mice, or by means of a pump with an infusion rate of 0.2 $\mu \mathrm{l} / \mathrm{min}$ in rats. After delivery, the needle was left in place for $5 \mathrm{~min}$ before retraction.

For mice, 10-12-week-old mice were injected in SNpc (stereotaxic coordinates from bregma: AP -2.7 mm, $\mathrm{ML}+1.5 \mathrm{~mm}$, DV $-4.2 \mathrm{~mm}$ ) with AAV6 carrying the BiFC constructs (listed in Table S2) expressed under the control of the Synapsin 1 (Syn1) promoter, at a final injection titer of $1 \times 10^{12}$ genome copies $/ \mathrm{ml}$ $(\mathrm{gc} / \mathrm{ml})$. After 15 weeks, animals were sacrificed.

For rats, 8-10-week-old rats were injected in SNpc (stereotaxic coordinates from bregma: AP -5.3 mm, ML $-1.7 \mathrm{~mm}, \mathrm{DV}-7.2 \mathrm{~mm}$ ) with AAV6 carrying VN-aSyn and Tau WT-VC constructs expressed under the control of the Syn 1 promoter; $5 \times 10^{13} \mathrm{gc} / \mathrm{ml}$ were injected. Eight weeks after injection, animals were sacrificed.

\section{Cell Culture and Transfection}

\section{Human embryonic kidney 293 (HEK293) cell line}

HEK293 cells (\#85120602, Sigma-Aldrich) were cultured in Gibco DMEM supplemented with $10 \%$ Fetal Bovine Serum (FBS) and 1\% Penicillin-Streptomycin (P/S), all from Thermo Fisher Scientific, in a humidified atmosphere at $5 \% \mathrm{CO}_{2}$ at $37^{\circ} \mathrm{C}$. For immunocytochemistry, cells were plated in a 48-well plate, and $0.1 \mu \mathrm{g}$ of the corresponding DNA was delivered per well along with $1 \mu \mathrm{l}$ of Lipofectamine 2000 (\#11668019, Thermo Fisher Scientific), following the manufacturer's protocol. HEK293 cells were transfected at $60-70 \%$ confluency, and $24 \mathrm{~h}$ post-transfection they were fixed with $4 \%$ paraformaldehyde (PFA) for $15 \mathrm{~min}$. For western blot, cells were plated in a 12-well plate, and $0.4 \mu \mathrm{g}$ of DNA was delivered per well along with $4 \mu \mathrm{l}$ of Lipofectamine 2000. After $24 \mathrm{~h}$ cells were lysed.

\section{Human neuroblastoma (SH-SY5Y) cell line}

SH-SY5Y cells (\#94030304, Sigma-Aldrich) were grown in Gibco DMEM supplemented with $10 \%$ FBS and $1 \% \mathrm{P} / \mathrm{S}$. Cells were incubated in a humidified atmosphere at $5 \% \mathrm{CO}_{2}$ at $37^{\circ} \mathrm{C}$. For differentiation, plates were coated with collagen $G(1: 20 ; L 7213$, Biochrom) for 2 h, and cells plated at a concentration of 10.526 cells per $\mathrm{cm}^{2}$. The following 4 days cells were incubated in FBS free medium supplemented with $10 \mu \mathrm{M}$ retinoic acid (R2625, Sigma-Aldrich). After that, cells were incubated in FBS free medium supplemented with $50 \mathrm{ng} / \mathrm{ml}$ BDNF (212-GD, R\&D Systems). For immunocytochemistry, cells were plated in a 48-well plate, and $0.1 \mu \mathrm{g}$ of the corresponding DNA was delivered per well along with $1 \mu \mathrm{l}$ of Lipofectamine 2000, following the manufacturer's protocol. SH-SY5Y cells were transfected, and live-cell imaged every $30 \mathrm{~min}$ for $24 \mathrm{~h}$ when evaluating the time-dependent interaction of the BiFC constructs. For quantification of the ratio of BiFC positive cells, SH-SY5Y were fixed $24 \mathrm{~h}$ post-transfection.

\section{Western blot}

HEK293 were co-transfected with different combinations of BiFC constructs (Table S1). After 24 h, cells were lysed in Pierce RIPA buffer (\#89900, Thermo Fisher Scientific) containing protease inhibitor (1:100; 
\#78430, Thermo Fisher Scientific) and phosphatase inhibitor (1:100; \#11833955, Thermo Fisher Scientific). Protein concentration was measured by BCA assay (\#23227, Thermo Fisher Scientific), and 5 $\mu \mathrm{g}$ of protein per sample were denatured at $95^{\circ} \mathrm{C}$ for $5 \mathrm{~min}$. Proteins were separated in NuPAGE 4-12\% BisTris gels (\#NP0321PK2, Thermo Fisher Scientific), and then transferred to nitrocellulose membranes (\#IB23001, Invitrogen) using an iBlot 2 gel transfer device (Invitrogen). Membranes were blocked in 5\% Bovine Serum Albumin (BSA; \#A7906, Sigma-Aldrich) for $1 \mathrm{~h}$ and incubated overnight in primary antibodies against aSyn, total Tau or $\beta$-Actin (Table S3) overnight. After that, membranes were washed in PBS with $0.05 \%$ Tween (PBS-T), incubated in anti-mouse or anti-rabbit horseradish peroxidase (HRP) secondary antibody for $1 \mathrm{~h}$, washed in PBS-T, incubated in enhanced chemiluminescence (ECL) substrate (\#1705061, BioRad) and developed in a ChemiDoc MP imager (BioRad).

\section{Live-Cell Imaging and Quantification}

After 5 days of differentiation, SH-SY5Y cells were (co-)transfected with different combinations of BiFC constructs (Table S1). After transfection, Venus complementation in SH-SY5Y cells was recorded by livecell imaging every $30 \mathrm{~min}$ for $24 \mathrm{~h}$. Images were taken in a Nikon Eclipse Ti microscope under a 10x objective. For each condition, a field of view (FOV) was chosen and tracked, changes in fluorescence intensity in the cells within the FOV with time were measured. The corrected fluorescence was calculated and divided within the total amount of BiFC positive cells present in the FOV after the $24 \mathrm{~h}$ :

\section{Total fluorescence - Initial background fluorescence}

\section{Total BiFC positive cells}

Alternatively, $24 \mathrm{~h}$ after transfection, SH-SY5Y cells were fixed with 4\% PFA for $15 \mathrm{~min}$, washed with PBST, incubated in DAPI (1:1000; D9542, Sigma-Aldrich) diluted in PBS for 30 min and washed again in PBST. Image acquisition was performed using an inverted epifluorescence microscope Olympus BX53, under a 20x objective. After that, the ratio of BiFC positive cells per total amount of cells in the FOV was quantified using Metamorph Premier (Cairn Research Ltd, Kent, UK).

\section{Immunohistochemistry}

Animals were deeply anaesthetized with isoflurane, perfused first with PB-buffer, followed by perfusion with 4\% PFA, and the brains extracted. Brains were post-fixed overnight and stored in 0.1 M PBS with 30\% sucrose until sectioned. For mouse brain samples, $40 \mu \mathrm{m}$ sections were mounted in polyvinyl alcohol mounting medium with DABCO (PVA-DABCO) containing DAPI (1:1000). Venus expression was acquired by using an inverted epifluorescence microscope Nikon Eclipse 80i under a $4 x$ objective, and a Leica SP8 confocal microscope under a 40x objective.

For rat brain samples, $40 \mu \mathrm{m}$ sections were washed in PBS-T, quenched in methanol and $\mathrm{H}_{2} \mathrm{O}_{2}(9: 1)$, washed in PBS-T, blocked in 5\% BSA for $1 \mathrm{~h}$, and incubated in aSyn (clone 211) or Tau-5 primary antibodies overnight. After incubation, sections were washed in PBS-T and incubated in anti-mouse HRP secondary antibody for $2 \mathrm{~h}$, washed in PBS-T, incubated in ABC-solution (\#PK-6100, Vector Laboratories), 
and washed in PBS-T and incubated in DAB solution (\#SK-4100, Vector Laboratories). After that, sections were dehydrated and mounted in dibutyl phthalate in xylene (DPX; \#06522, Sigma-Aldrich). For immunofluorescence, the sections were rinsed in TBS, pre-incubated in 10\% Normal Donkey Serum (NDS) for $1 \mathrm{~h}$ and incubated overnight in pS129 and AT8 primary antibodies. Next, sections were rinsed with TBS, incubated in anti-rabbit and anti-mouse secondary antibodies for $1 \mathrm{~h}$, rinsed with TBS and mounted in PVA-DABCO. After DAB staining, sections were scanned using an EPSON perfection V750 PRO with Silver Fast software. Fluorescence images were acquired in an Olympus BX53 microscope under a 10x objective. See list of antibodies in Table S3.

\section{Statistical analyses}

Data analysis was performed using GraphPad Prism v.9.2. Data were expressed as mean \pm SD unless stated otherwise. Kruskal-Wallis test was performed to compare the ratio of BiFC positive cells per total amount of cells in FOV obtained $24 \mathrm{~h}$ after (co-) transfection of SH-SY5Y cells. Data was considered statistically significant when $p$-value $<0.05\left({ }^{*} p<0.05, * * p<0.01\right)$.

The datasets generated during the current study are available from the corresponding authors on reasonable request.

\section{Declarations}

\section{ACKNOWLEDGEMENTS}

The authors would like to thank to Alicja Flasch for her technical support. We also thank Janitha M. Mudannayake and Sabine C. Konings, Lund University, for their feedback on this manuscript. We acknowledge the Strategic Research Environment MultiPark for providing some of the infrastructure used in this work.

\section{Funding}

This project was supported by the EU Horizon 2020 Marie Sklodowska-Curie innovative training network SynDegen (grant agreement No 721802). J-Y.L. is supported by the Swedish Research Council (201901551); ParkinsonFonden; AlzheimerFonden; the Strategic Research Area Multipark (Multidisciplinary research in Parkinson's disease at Lund University); and EU-JPND research (aSynProtec and REfrAME). G.K.G. is supported by the Swedish Research Council (2019-01125) and the Strategic Research Area Multipark. T.F.O. is supported by the Deutsche Forschungsgemeinschaft (DFG, German Research Foundation) under Germany's Excellence Strategy - EXC 2067/1- 390729940, and by SFB1286 (B8).

\section{AUTHOR CONTRIBUTIONS}

J-Y.L., G.K.G., and T.F.O. conceived the idea and supervised the project; L.T.G. and J.M.P.D. designed the experiments; J.M.P.D., E.B. and W.L. injected and sacrificed the animals; J.M.P.D., L.T.G., I.C.B., E.G., and 
A.S. designed and characterized the BiFC constructs; L.T.G., J.M.P.D., and C.H. performed live-cell imaging and microscopy; L.T.G. wrote the manuscript. All the authors reviewed and approved the final manuscript.

\section{Competing Interests}

The authors have no competing interests to declare.

\section{References}

1. Duka, T. et al. Alpha-Synuclein induces hyperphosphorylation of Tau in the MPTP model of Parkinsonism. FASEB J, 20, 2302-2312 (2006).

2. Arriagada, P. V., Growdon, J. H., Hedley-Whyte, E. T. \& Hyman, B. T. Neurofibrillary tangles but not senile plaques parallel duration and severity of Alzheimer's disease. Neurology, 42, 631 (1992).

3. Martin, E. R. Association of Single-Nucleotide Polymorphisms of the Tau Gene With Late-Onset Parkinson Disease. JAMA, 286, 2245 (2001).

4. Edwards, T. L. et al. Genome-Wide Association Study Confirms SNPs in SNCA and the MAPT Region as Common Risk Factors for Parkinson Disease. Ann. Hum. Genet, 74, 97-109 (2010).

5. Hamilton, R. L. Lewy Bodies in Alzheimer's Disease: A Neuropathological Review of 145 Cases Using a-Synuclein Immunohistochemistry. Brain Pathol, 10, 378-384 (2006).

6. Spires-Jones, T. L., Attems, J. \& Thal, D. R. Interactions of pathological proteins in neurodegenerative diseases. Acta Neuropathol. (Berl.), 134, 187-205 (2017).

7. Wilhelmsen, K. C. et al. 17q-Linked Frontotemporal Dementia-Amyotrophic Lateral Sclerosis Without Tau Mutations With Tau and a-Synuclein Inclusions. Arch. Neurol, 61, 398 (2004).

8. Yancopoulou, D., Xuereb, J. H., Crowther, R. A., Hodges, J. R. \& Spillantini, M. G. Tau and a-Synuclein Inclusions in a Case of Familial Frontotemporal Dementia and Progressive Aphasia. J. Neuropathol. Exp. Neurol, 64, 245-253 (2005).

9. Uchikado, H., DelleDonne, A., Uitti, R. \& Dickson, D. W. Coexistence of PSP and MSA: a case report and review of the literature. Acta Neuropathol. (Berl.), 111, 186-192 (2006).

10. Raghavan, R. et al. Detection of Lewy Bodies in Trisomy 21 (Down's Syndrome). Can. J. Neurol. Sci. J. Can. Sci. Neurol, 20, 48-51 (1993).

11. Lippa, C. F., Schmidt, M. L., Lee, V. M. Y. \& Trojanowski, J. Q. Antibodies to a-synuclein detect Lewy bodies in many Down's syndrome brains with Alzheimer's disease. Ann. Neurol, 45, 353-357 (1999).

12. Schmidt, M. L., Martin, J. A., Lee, V. M. Y. \& Trojanowski, J. Q. Convergence of Lewy bodies and neurofibrillary tangles in amygdala neurons of Alzheimer's disease and Lewy body disorders. Acta Neuropathol. (Berl.), 91, 475-481 (1996).

13. Colom-Cadena, M. et al. Confluence of a-Synuclein, Tau, and $\beta$-Amyloid Pathologies in Dementia With Lewy Bodies. J. Neuropathol. Exp. Neurol, 72, 1203-1212 (2013).

14. Sengupta, U. et al. Pathological Interface Between Oligomeric Alpha-Synuclein and Tau in Synucleinopathies. Biol. Psychiatry, 78, 672-683 (2015). 
15. Jellinger, K. A. \& Attems, J. Prevalence and impact of vascular and Alzheimer pathologies in Lewy body disease. Acta Neuropathol. (Berl.), 115, 427-436 (2008).

16. Chin, K. S., Yassi, N., Churilov, L., Masters, C. L. \& Watson, R. Prevalence and clinical associations of tau in Lewy body dementias: A systematic review and meta-analysis. Parkinsonism Relat. Disord, 80, 184-193 (2020).

17. Lippa, C. F. et al. Lewy Bodies Contain Altered a-Synuclein in Brains of Many Familial Alzheimer's Disease Patients with Mutations in Presenilin and Amyloid Precursor Protein Genes. Am. J. Pathol, 153, 1365-1370 (1998).

18. Marui, W., Iseki, E., Uéda, K. \& Kosaka, K. Occurrence of human a-synuclein immunoreactive neurons with neurofibrillary tangle formation in the limbic areas of patients with Alzheimer's disease. $J$. Neurol. Sci, 174, 81-84 (2000).

19. Bassil, F. et al. a-Synuclein modulates tau spreading in mouse brains. J. Exp. Med, 218, e20192193 (2021).

20. Teravskis, P. J. et al. A53T Mutant Alpha-Synuclein Induces Tau-Dependent Postsynaptic Impairment Independently of Neurodegenerative Changes. J. Neurosci, 38, 9754-9767 (2018).

21. Singh, B. et al. Tau is required for progressive synaptic and memory deficits in a transgenic mouse model of a-synucleinopathy. Acta Neuropathol. (Berl.), 138, 551-574 (2019).

22. Klein, R., Dayton, R., Lin, W. \& Dickson, D. Tau gene transfer, but not alpha-synuclein, induces both progressive dopamine neuron degeneration and rotational behavior in the rat. Neurobiol. Dis, 20,6473 (2005).

23. Ittner, L. M. et al. Parkinsonism and impaired axonal transport in a mouse model of frontotemporal dementia. Proc. Natl. Acad. Sci. 105, 15997-16002(2008).

24. Badiola, N. et al. Tau Enhances a-Synuclein Aggregation and Toxicity in Cellular Models of Synucleinopathy. PLoS ONE, 6, e26609 (2011).

25. Jensen, P. H. et al. a-Synuclein Binds to Tau and Stimulates the Protein Kinase A-catalyzed Tau Phosphorylation of Serine Residues 262 and 356. J. Biol. Chem, 274, 25481-25489 (1999).

26. Stoyka, L. E. et al. Templated a-Synuclein Inclusion Formation Is Independent of Endogenous Tau. eneuro 8, ENEURO.0458-20.2021(2021).

27. Dasari, A. K. R., Kayed, R., Wi, S. \& Lim, K. H. Tau Interacts with the C-Terminal Region of a-Synuclein, Promoting Formation of Toxic Aggregates with Distinct Molecular Conformations., 58, 2814-2821 (2019).

28. Outeiro, T. F. et al. Formation of Toxic Oligomeric a-Synuclein Species in Living Cells. PLoS ONE, 3, e1867 (2008).

29. Lázaro, D. F. et al. Systematic Comparison of the Effects of Alpha-synuclein Mutations on Its Oligomerization and Aggregation. PLoS Genet, 10, e1004741 (2014).

30. Eckermann, K., Kügler, S. \& Bähr, M. Dimerization propensities of Synucleins are not predictive for Synuclein aggregation. Biochim. Biophys. Acta BBA - Mol. Basis Dis, 1852, 1658-1664 (2015). 
31. Dominguez-Meijide, A. et al. Effects of pharmacological modulators of a-synuclein and tau aggregation and internalization. Sci. Rep, 10, 12827 (2020).

32. Frey, B. et al. Monitoring alpha-synuclein oligomerization and aggregation using bimolecular fluorescence complementation assays: What you see is not always what you get. J. Neurochem. jnc, 15147, https://doi.org/10.1111/jnc.15147 (2020).

33. Tak, H. et al. Bimolecular Fluorescence Complementation; Lighting-Up Tau-Tau Interaction in Living Cells. PLOS ONE, 8, e81682 (2013).

34. Herrera, F., Tenreiro, S., Miller-Fleming, L. \& Outeiro, T. F. Visualization of cell-to-cell transmission of mutant huntingtin oligomers. PLoS Curr, 3, RRN1210 (2011).

35. Blum, D. et al. Mutant huntingtin alters Tau phosphorylation and subcellular distribution. Hum. Mol. Genet, 24, 76-85 (2015).

36. Repici, M. et al. Parkinson's disease-associated mutations in DJ-1 modulate its dimerization in living cells. J. Mol. Med, 91, 599-611 (2013).

37. Zondler, L. et al. DJ-1 interactions with a-synuclein attenuate aggregation and cellular toxicity in models of Parkinson's disease. Cell Death Dis, 5, e1350-e1350 (2014).

38. Foglieni, C. et al. Split GFP technologies to structurally characterize and quantify functional biomolecular interactions of FTD-related proteins. Sci. Rep, 7, 14013 (2017).

39. Cai, W., Feng, D., Schwarzschild, M. A., McLean, P. J. \& Chen, X. Bimolecular Fluorescence Complementation of Alpha-synuclein Demonstrates its Oligomerization with Dopaminergic Phenotype in Mice. EBioMedicine, 29, 13-22 (2018).

40. Kiechle, M. et al. In Vivo Protein Complementation Demonstrates Presynaptic a-Synuclein Oligomerization and Age-Dependent Accumulation of 8-16-mer Oligomer Species. Cell Rep, 29, 2862-28749 (2019).

41. Shin, S. et al. Visualization of soluble tau oligomers in TauP301L-BiFC transgenic mice demonstrates the progression of tauopathy. Prog. Neurobiol, 187, 101782 (2020).

42. Burré, J., Sharma, M. \& Südhof, T. C. Cell Biology and Pathophysiology of a-Synuclein. Cold Spring Harb. Perspect. Med, 8, a024091 (2018).

43. Wang, Y. \& Mandelkow, E. Tau in physiology and pathology. Nat. Rev. Neurosci, 17, 22-35 (2016).

44. Guo, T., Noble, W. \& Hanger, D. P. Roles of tau protein in health and disease. Acta Neuropathol. (Berl.), 133, 665-704 (2017).

45. Weinreb, P. H. et al. A Protein Implicated in Alzheimer's Disease and Learning, Is Natively Unfolded., 35, 13709-13715 (1996).

46. Mukrasch, M. D. et al. Structural Polymorphism of 441-Residue Tau at Single Residue Resolution. PLOS Biol, 7, e1000034 (2009).

47. Brás, I. C. et al. Common molecular mechanisms underlie the transfer of alpha-synuclein, Tau and huntingtin and modulate spontaneous activity in neuronal cells. http://biorxiv.org/lookup/doi/10.1101/2021.07.18.452825 (2021) doi:10.1101/2021.07.18.452825. 
48. Goedert, M., Eisenberg, D. S. \& Crowther, R. A. Propagation of Tau Aggregates and Neurodegeneration. Annu. Rev. Neurosci, 40, 189-210 (2017).

49. Robida, A. M. \& Kerppola, T. K. Bimolecular Fluorescence Complementation Analysis of Inducible Protein Interactions: Effects of Factors Affecting Protein Folding on Fluorescent Protein Fragment Association. J. Mol. Biol, 394, 391-409 (2009).

50. Hernandez, F. P. \& Sandri-Goldin, R. M. Head-to-Tail Intramolecular Interaction of Herpes Simplex Virus Type 1 Regulatory Protein ICP27 Is Important for Its Interaction with Cellular mRNA Export Receptor TAP/NXF1. mBio 1, e00268-10(2010).

51. Hu, C. D. \& Kerppola, T. K. Simultaneous visualization of multiple protein interactions in living cells using multicolor fluorescence complementation analysis. Nat. Biotechnol, 21, 539-545 (2003).

52. Hu, C. D., Chinenov, Y. \& Kerppola, T. K. Visualization of Interactions among bZIP and Rel Family Proteins in Living Cells Using Bimolecular Fluorescence Complementation. Mol. Cell, 9, 789-798 (2002).

53. Braak, H. \& Braak, E. Neuropathological stageing of Alzheimer-related changes. Acta Neuropathol. (Berl.), 82, 239-259 (1991).

54. Braak, H. et al. Staging of brain pathology related to sporadic Parkinson's disease. Neurobiol. Aging, 24, 197-211 (2003).

55. Liu, L. et al. Trans-Synaptic Spread of Tau Pathology In Vivo. PLoS ONE, 7, e31302 (2012).

56. Schaser, A. J. et al. Trans-synaptic and retrograde axonal spread of Lewy pathology following preformed fibril injection in an in vivo A53T alpha-synuclein mouse model of synucleinopathy. Acta Neuropathol. Commun, 8, 150 (2020).

57. Franzmeier, N. et al. Tau spreading is driven by neuronal connectivity in primary tauopathies evidence from tau-PET and histopathology. http://medrxiv.org/lookup/doi/10.1101/2021.08.16.21261523 (2021) doi:10.1101/2021.08.16.21261523.

58. Kordower, J. H., Chu, Y., Hauser, R. A., Freeman, T. B. \& Olanow, C. W. Lewy body-like pathology in long-term embryonic nigral transplants in Parkinson's disease. Nat. Med, 14, 504-506 (2008).

59. Li, J. Y. et al. Lewy bodies in grafted neurons in subjects with Parkinson's disease suggest host-tograft disease propagation. Nat. Med, 14, 501-503 (2008).

60. Hoover, B. R. et al. Tau Mislocalization to Dendritic Spines Mediates Synaptic Dysfunction Independently of Neurodegeneration., 68, 1067-1081 (2010).

\section{Figures}


A

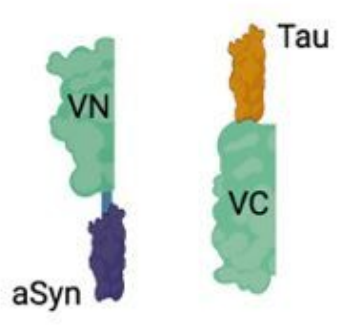

\section{B}
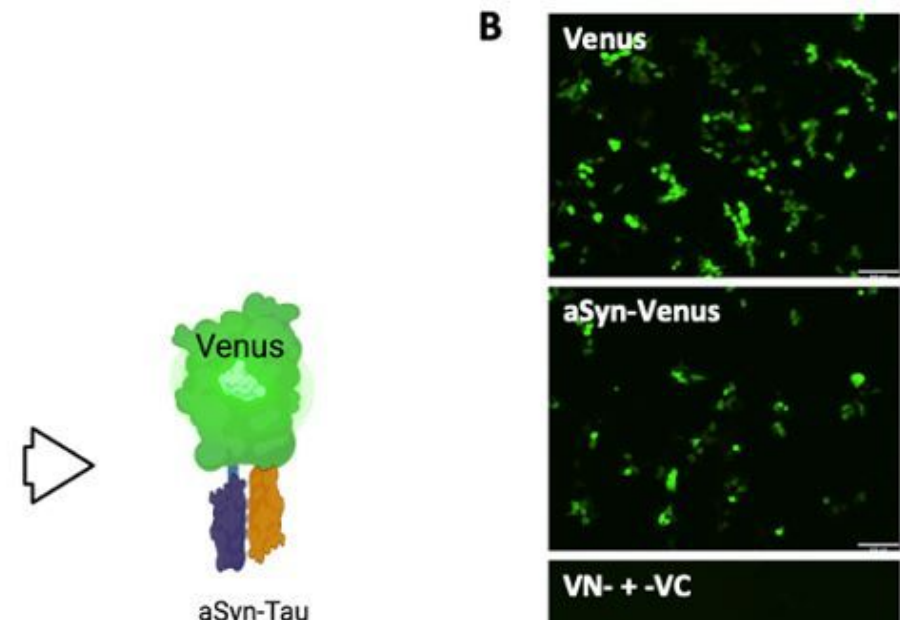

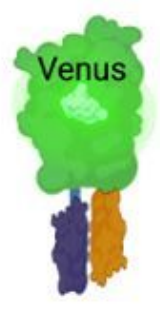

aSyn-Tau interaction
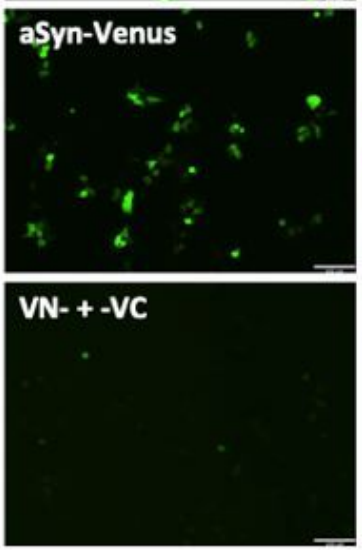

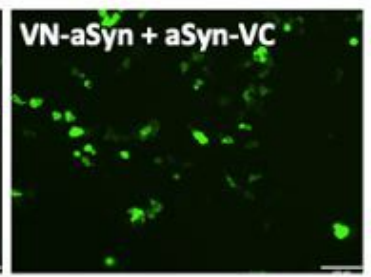

VN-Tau WT + Tau WT-VC
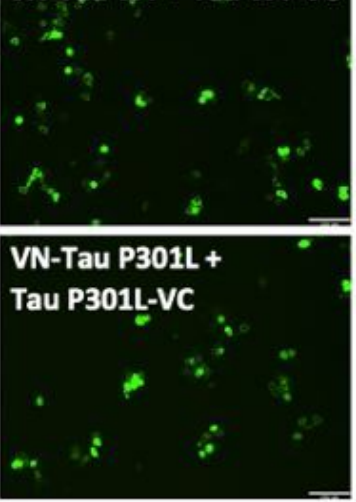

\section{C}
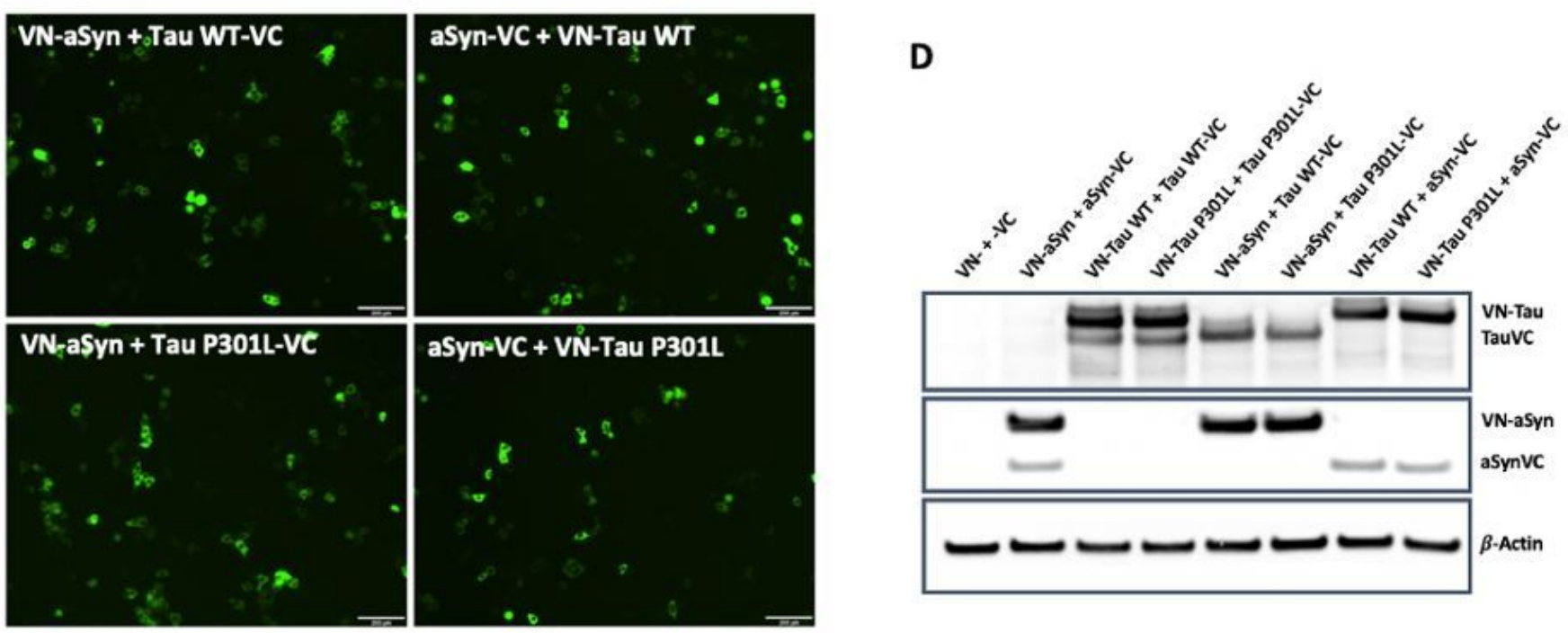

\section{Figure 1}

Bimolecular Fluorescence Complementation (BiFC) assay confirms aSyn-Tau interaction in HEK293 cells. A) Schematic representation of the BiFC assay. aSyn WT and/or Tau (WT or P301L) were expressed fused to one of the halves of the protein Venus (VN- or - VC). Complementation of the Venus halves due to interaction of aSyn with Tau promotes changes in the molecular conformation of the Venus protein leading to its emission of fluorescence (scheme created with BioRender.com). B) Fluorescence emitted by Venus complementation $24 \mathrm{~h}$ post-transfection in HEK293 cells shows self-interaction of aSyn, Tau WT and Tau P301L. HEK293 cells were transiently (co-)transfected with different combinations of the BiFC constructs; the fluorescence emitted by Venus expression or complementation was recorded $24 \mathrm{~h}$ after transfection. Scale bar: $200 \mu \mathrm{m}$. C) Fluorescence emitted by Venus complementation $24 \mathrm{~h}$ posttransfection in HEK293 cells shows interaction of aSyn with Tau (WT and P301L). HEK293 cells were 
transiently co-transfected with different combinations of the aSyn and Tau constructs, and the fluorescence emitted by Venus complementation was recorded $24 \mathrm{~h}$ after transfection. Scale bar: $200 \mu \mathrm{m}$. D) Total levels of Tau and aSyn measured by western blot (WB). The levels of expression of aSyn and Tau in the cell lysate of HEK293 cells co-transfected with different BiFC constructs were measured by WB against total Tau and aSyn. $\beta$-Actin was used as loading control.
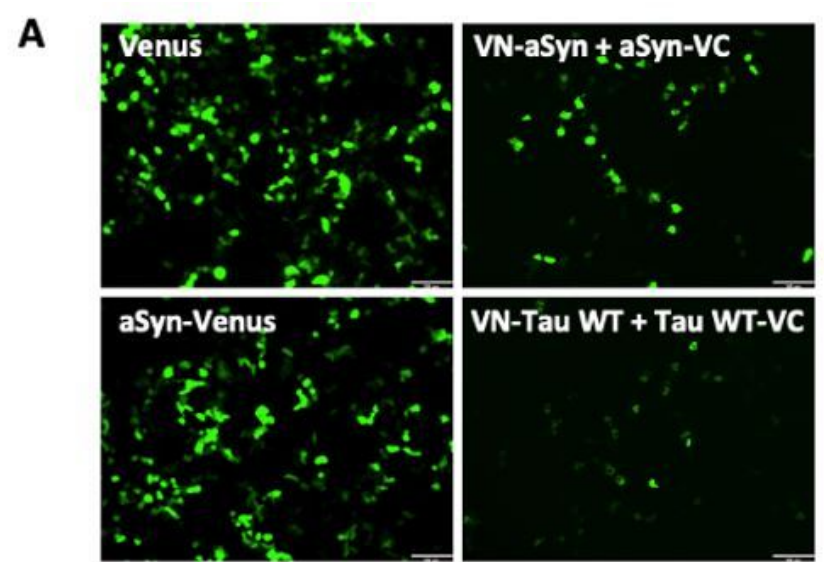

VN- + -VC

B
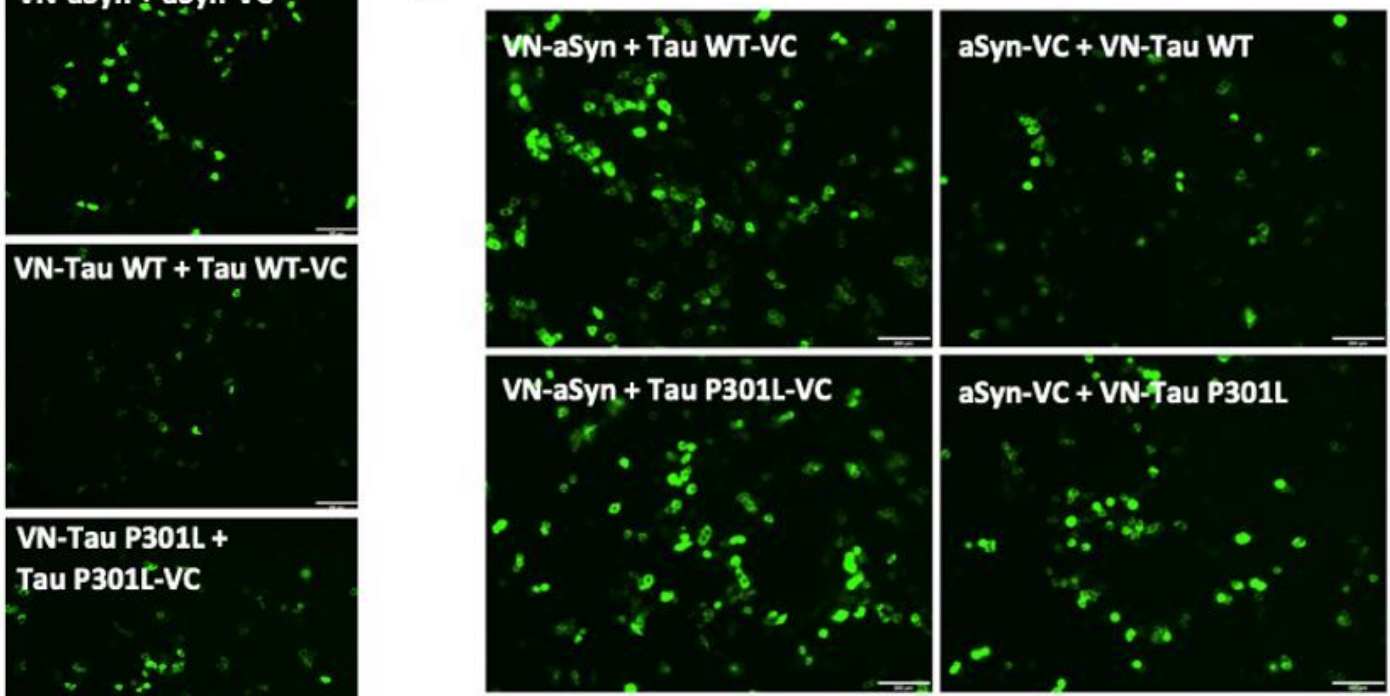

Tau P301E-vC

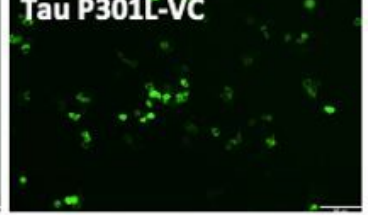

C

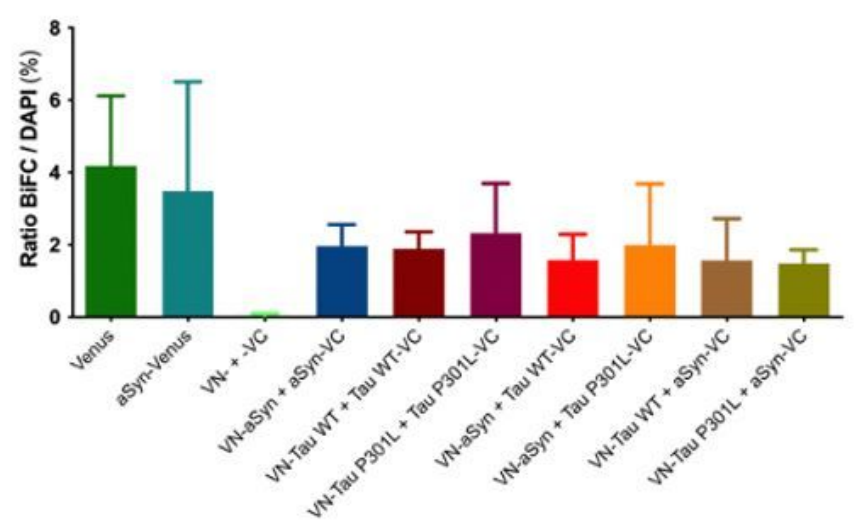

Figure 2

aSyn-Tau interaction in SH-SY5Y human neuroblastoma cells. A) Fluorescence emitted by Venus complementation $24 \mathrm{~h}$ post-transfection in SH-SY5Y cells shows self-interaction of aSyn, Tau WT and Tau P301L. SH-SY5Y cells were transiently (co-)transfected with different combinations of the BiFC constructs, and the fluorescence emitted by Venus expression or complementation was recorded $24 \mathrm{~h}$ after transfection. Scale bar: $200 \mu \mathrm{m}$. B) Fluorescence emitted by Venus complementation $24 \mathrm{~h}$ posttransfection in SH-SY5Y cells shows interaction of aSyn with Tau (WT and P301L). SH-SY5Y cells were 
transiently co-transfected with different combinations of the aSyn and Tau construct, and the fluorescence emitted by Venus complementation was recorded $24 \mathrm{~h}$ after transfection. Scale bar: $200 \mu \mathrm{m}$. C) BiFC interaction in (co-)transfected SH-SY5Y cells after $24 \mathrm{~h}$. The percentage of fluorescent (BiFC positive) SH-SY5Y cells in relation to the total number of cells present in the FOV was quantified. Data are expressed as mean \pm SD. $\mathrm{n}=3$.

\section{$B / F$ \\ Venus}
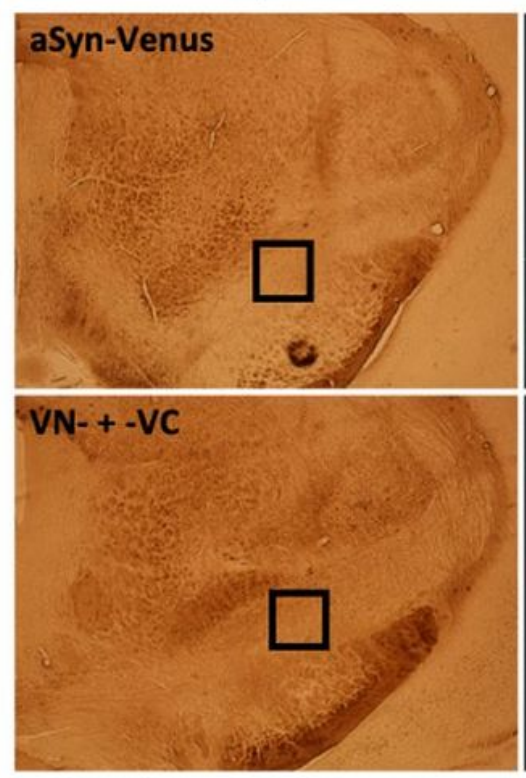

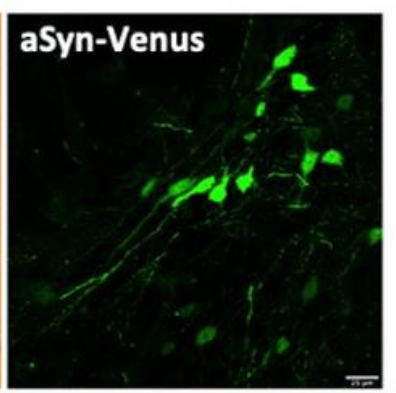

VN-+-VC
$\mathbf{B} / \mathbf{F}$

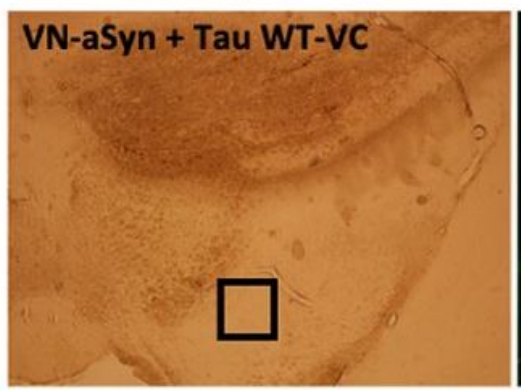

aSyn-VC+ VN-Tau WT

0

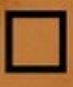

Venus

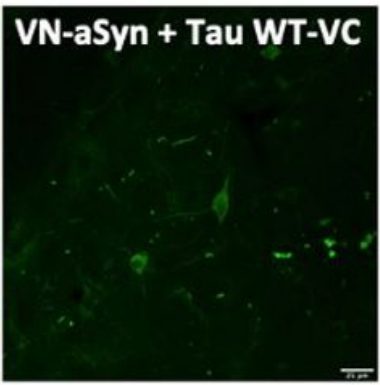

aSyn-VC + VN-Tau WT

\section{Figure 3}

aSyn-Tau interaction in mouse substantia nigra pars compacta (SNpc). BiFC interaction in mouse SNpc. 10-12-week-old mice were injected in SNpc with AAV6 carrying different BiFC constructs. After 15 weeks, Venus fluorescence was evaluated. The expression of aSyn fused to full-length Venus provided the maximum level of fluorescence expected. The interaction of aSyn and Tau was reflected by Venus complementation in VN-aSyn + Tau WT-VC and VN-Tau WT + aSyn-VC injected animals. As expected, the expression of the Venus halves not linked to proteins did not produce any fluorescence in the injected area. Boxed area on Bright Field (B/F) images is magnified to the right. Scale bar: $25 \mu \mathrm{m}$. 

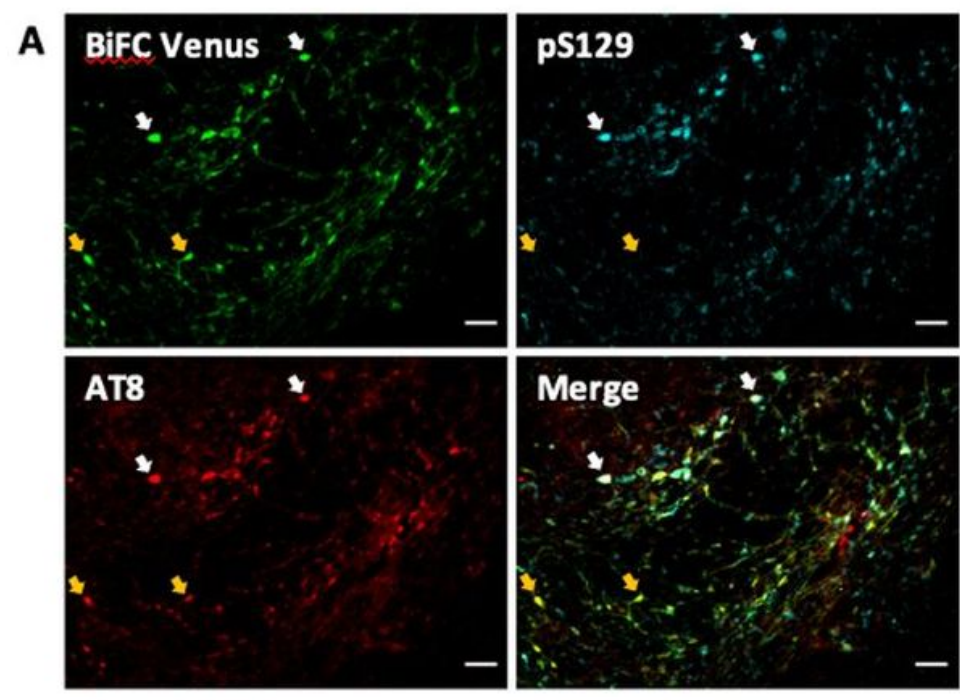

B

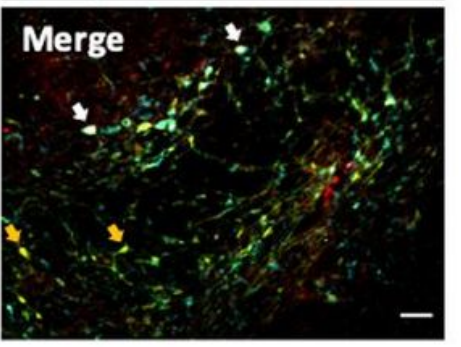

aSyn (211)

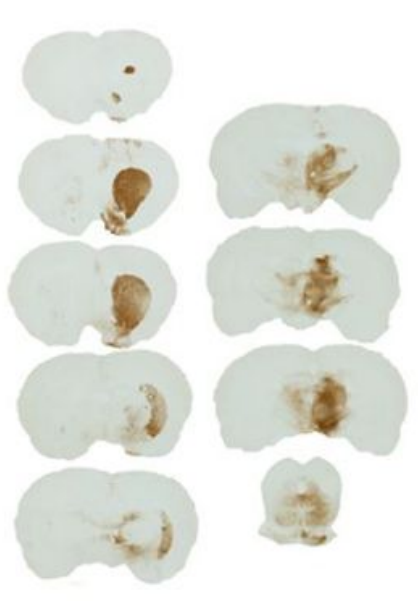

Tau (Tau-5)

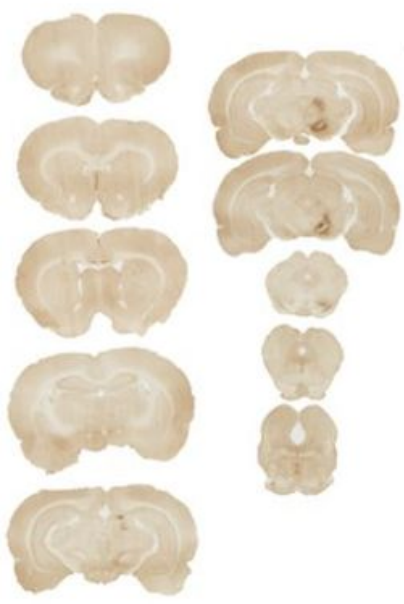

\section{Figure 4}

Molecular characterization of aSyn-Tau interaction in rat SNpc. aSyn-Tau interaction in rat SNpc. Eight weeks after co-injection of VN-aSyn and Tau WT-VC in rat SNpc immunohistochemistry was performed. A) Immunohistochemistry for phosphorylated epitopes of aSyn (pS129) and Tau (AT8) suggests that aSyn and Tau can be phosphorylated while they are interacting with each other. B) Distribution of aSyn and Tau after injection of VN-aSyn and Tau WT-VC in rat SNpc. aSyn distribution shows a marked spread of aSyn from the injected area to the striatum. In contrast, the expression of human Tau seems to be confined to the SNpc. Scale bar: $50 \mu \mathrm{m}$

\section{Supplementary Files}

This is a list of supplementary files associated with this preprint. Click to download.

- LauraTGTauaSynSuppMaterial.pdf 